\title{
Investigations of families of patients diagnosed with gastric carcinoma in Bulgaria
}

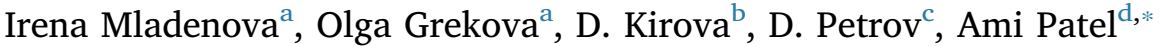 \\ a Trakia University, Medical Faculty, Dept. of Epidemiology and Infectious Diseases, Stara Zagora, Bulgaria \\ b Complex Oncology Center - Stara Zagora EOOD, Stara Zagora, Bulgaria \\ ${ }^{\mathrm{c}}$ Trakia University, Medical Faculty, Dept. of Social Medicine, Stara Zagora, Bulgaria \\ ${ }^{\mathrm{d}}$ Division of Dairy and Food Microbiology, Mansinhbhai Institute of Dairy \& Food Technology-MIDFT, Dudhsagar Dairy campus, Mehsana 384 002, Gujarat, India
}

\section{A R T I C L E I N F O}

\section{Keywords:}

H. pylori- infection

Gastric carcinoma

Epidemiology

Risk factors

\begin{abstract}
A B S T R A C T
Background: To identify the prevalence of $H$. pylori in patients with gastric carcinoma and their first-degree relatives, the possible risks of acquisition of infection and developing of gastric cancer.

Methods: We investigated 31 families (total 75 human volunteers) with a family member, diagnosed as a gastric carcinoma patient and their first-degree relatives. Patients were tested forH. pylori stool antigen by "DIAQUICK" H. pylori Stool Cassette- a rapid test for the qualitative detection of $H$. pylori antigen. A questionnaire for sociodemographic and lifestyle possible risk factors for acquisition of infection and for developing of gastric cancer was completed by 75 people. The statistical analysis was performed using Fisher's test and ANOVA.

Results: Fisher's Test shows that smoking $\left(\chi^{2}=4.172 ; \mathrm{p}=0.386\right)$, animals at home $(\chi 2=0.376 ; \mathrm{p}=0.871)$, and diet $\left(\chi^{2}=2.166 ; \mathrm{p}=0.725\right)$ are not significantly associated; however, the family history of gastric cancer $\left(\chi^{2}=56.886 ; \mathrm{p}=0.001\right)$, level of education of patients $\left(\chi^{2}=17.374 ; \mathrm{p}=0.004\right)$ and $\operatorname{sex}\left(\chi^{2}=6.669\right.$; $\mathrm{p}=0.039$ ) are significantly associated with the acquisition of infection and the developing of gastric cancer in patients and their first-degree relatives.

Conclusion: Concern authorities should develop and implement strategies to improve educational status, basic sanitary facilities, and socioeconomic status to minimize $H$. pylori infection.
\end{abstract}

\section{Introduction}

Helicobacter pylori (H. pylori) is a spiral shaped gram negative, nonspore forming bacterium which colonizes the human stomach. Basically, H. pylori is a highly prevalent pathogen associated with chronic gastritis and peptic ulcer, and is a risk factor for gastric malignancies. ${ }^{1}$ Moreover, the bacterium is also thought to be involved in other human illnesses like autoimmune disorders, ${ }^{2}$ insulin resistance ${ }^{3}$ and the metabolic syndrome. ${ }^{4}$ In 1994, on the basis of epidemiological studies, the International Agency for Research on Cancer identified $H$. pylori as a Group 1 carcinogen. ${ }^{5}$ Helicobacter pylori is strongly associated with gastric carcinoma and MALT- lymphoma. Risk of non-cardia gastric cancer is six times higher for $H$. pylori- infected people than for uninfected people. Gastric cancer is the second most common cause of cancer-related death in the world. The worldwide incidence of gastric cancer has declined over the recent decades, due to the recognition of risk factors such as $H$. pylori and other dietary and environmental risks. ${ }^{6,7}$

One meta-analysis has demonstrated that first-degree relatives of patients with gastric cancer might be at an increased risk of developing gastric cancer by significantly higher prevalence of $H$. pylori, gastric atrophy and IM (intestinal metaplasia), in comparison with controls. ${ }^{8}$ Shin et al. ${ }^{9}$ have compared uninfected subjects without a family history of gastric cancer and subjects with both a family history and $H$. pylori infection. The results have been shown a 5-fold increased risk of gastric cancer (OR 5.32, 95\% CI: 2.76-10.25). ${ }^{9}$ The level of education is also one of the prime factors associated with gastric cancer. Several studies have indicated that individuals with lower educational levels had a higher risk than those with a higher education. ${ }^{10,11}$ In addition to this, Eusebi et al. ${ }^{12}$ confirmed that one of the most important factors to acquired $H$. pyloriinfection is low socioeconomic conditions in childhood.

In Bulgaria, stomach cancer is the sixth most common cancer in men (5.5\%) and the eighth - in women (4.1\%). It accounts for $4.8 \%$ of all cancer cases. In 2012, there were 1553 new cancer cases, of which $60.9 \%$ were in men. The crude incidence rates were 26.6 per 100,000 men and 16.2 per 100,000 women. In 2012, there were 1288 stomach cancer deaths, of which $61.6 \%$ were in men. The crude mortality rates were 22.3 per 100,000 men and 13.2 per 100,000 women. More than

\footnotetext{
* Corresponding author

E-mail address: ami@midft.com (A. Patel).
} 
half of patients (53.2\%) were diagnosed in the later stages of the disease ( 3 and 4 th stage); $23.7 \%$ on the 1 st and the 2 nd stage, while nearly $25 \%$ - unclear stage.76.4\% of the new cases were morphologically confirmed and in the majority of them the diagnosis was adenocarcinoma. ${ }^{13}$ Based on such data, the estimates for expected new stomach cancer cases in 2016 are 1414 (95\% confidence interval: from 1238 to 1590) and for expected cancer deaths, 1107 (95\% confidence interval: from 929 to 1285$){ }^{14}$

Investigating the epidemiological aspects of H.pylori infection is imperative and it would serve to control as well as reduce the consequences and complications related with the infection. Further, it is fundamental for developing eradication treatment and diminished the incidences of antibiotic resistance. ${ }^{15}$ The aim of this study is to identify the prevalence of H.pylori in patients with gastric carcinoma and their first-degree relatives, the possible risks of acquisition of infection and the developing of gastric cancer, in the region of the Stara Zagora, Bulgaria.

\section{Methods}

We investigated 31 families with a family member, diagnosed as a gastric carcinoma patient and their first-degree relatives (total 75 people). The patients were tested for $H$. pylori stool antigen by "DIAQUICK" H. pylori Stool Cassette- a rapid, one step, and visual immunechromatographic test for the qualitative detection of $H$. pylori antigen in faecal samples. The membrane is coated with monoclonal antibodies against $H$. pylori, in the field of the test strip. During testing the sample reacts with the coloured conjugate previously dried on the strip. By capillary road the complexes move on the membrane. In the case of a positive result the specific antibodies present on the membrane are contacted with the coloured conjugate.

A questionnaire (consisting total 38 questions) for sociodemographic and lifestyle possible risk factors for acquisition of infection and for the developing of gastric cancer was completed by 75 people. For statistical analysis were used: Fischer test (Fisher's Exact Test), Levin test (Levine), post-hoc Bonferroni test (Bonferonni) and ANOVA (analysis of variance).

\section{Results}

The mean age of patients with gastric cancer is $(69.25 \pm 10.526)$ and the mean age of their first-degree relatives (44.84 \pm 14.25) (Fig.1). Fisher's test (Fisher's Exact Test) has shown that the presence of pets $(\chi 2=0.376 ; \quad p=0.871)$, eating from common dishes $(\chi 2=2.166 ; p=0.725)$, the use of thermally unprocessed vegetables $(\chi 2=4.333 ; p=0.332)$, consumption of uncooked milk $(\chi 2=3.959$; $\mathrm{p}=0.399)$, and smoking $(\chi 2=4.172 ; \mathrm{p}=0.386)$ did not have a statistically significance (Table. 1$)$.

The sex of study participants $(\chi 2=6.669 ; \mathrm{p}=0.039$; Cramer's $\mathrm{V}=0,301)$, family history of stomach cancer $\left(\chi^{2}=56.886\right.$; $\mathrm{p}=0.001$; Cramer's $\mathrm{V}=0.6$ ), as well as the level of education $(\chi 2=17,374 ; \mathrm{p}=0.004$; Cramer's $\mathrm{V}=0,346)$ and residence in a rural area during the childhood ( $\chi 2=6.627 ; \mathrm{p}=0.036$, Cramer's $\mathrm{V}=0,3$ ) have moderate significance in terms of $H$. pylori- infection and

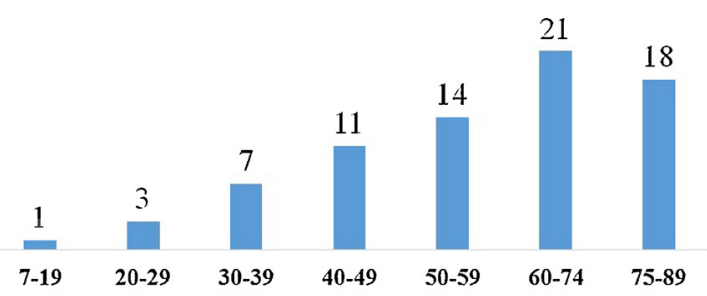

Fig. 1. Age distribution of patients.
Table 1

Possible risk factors for $H$. pylori infection.

\begin{tabular}{lll}
\hline Possible risk factors & $\chi^{2}$ & $\mathrm{p}$ \\
\hline Sex & 6.669 & 0.039 \\
Family history of gastric cancer & 56.886 & 0.001 \\
Level of education & 17.374 & 0.004 \\
Childhood living place & 6.627 & 0.036 \\
Smoking & 4.172 & 0.386 \\
Consumption of uncooked milk & 3.959 & 0.399 \\
Consumption of uncooked vegetables & 4.333 & 0.332 \\
Common eating dishes & 2.166 & 0.725 \\
Animals at home & 0.376 & 0.871 \\
\hline
\end{tabular}

development of gastric cancer-in patients and their first-degree relatives (Table.1). The differences can be explained. Outcomes of current study concord well with the findings of other investigations. ${ }^{10,11}$ The cancer patients are mostly elderly and minorities.

\section{Discussion}

A questionnaire was designed based on the previous studies of our group ${ }^{16,17}$ and related literature searches; it was mainly contained information regarding sociodemographic and lifestyle possible risk factors for acquisition of infection and for the developing of gastric cancer among the patients.

Foods which are not properly washed or sanitized usually harbour potential health hazarding compounds like pesticides, chemicals and/or microorganisms that may lead to life threatening diseases. In our study may be due to relatively small subjects, effect of uncooked vegetables or milk did not appear as a significant factors linked with the acquisition of infection with subsequent development of gastric cancer. Conversely, a recent meta-analysis including eight epidemiologic studies confirmed an increased risk of gastric cancer with a western or unhealthy diet/ food, in respect of a prudent/healthy diet such as fruits and vegetables (OR 1.51; 95\% CI 1.21-1.89). ${ }^{18}$ Similarly, recent reviews suggest that probiotics, health beneficiary bacteria (which mainly includes Lactobacillus and Bifidobacterium spp.) can combat with $H$. pylori by either immunological or non-immunological mechanisms. ${ }^{1,19}$ Thus, regular consumption of probiotics containing products in daily diet may help to cure $H$. pylori infection. Further, it would help to reduce the risk of $H$. pylori infection.

In current investigation, the family history of stomach cancer, sex of study participants, level of education and childhood living space seen to be the most significant factors associated with risk of acquiring infection and gastric cancer (Table 1). In this context, it is worth to mention that gastric cancer may not be associated hereditary in each case; however it cannot be excluded that the higher risk for gastric cancer seen among those with family history of gastric cancer might also be due to shared environmental factors like iron deficiency or high-salt diets diet and smoking. ${ }^{20}$ Such environmental factors may also have a significant effect during the childhood that can be linked with gastric cancer. The level of education can be linked with the awareness of people with importance of cleanliness and hygienic conditions at household as well as long term health hazards (such as cancer, tuberculosis, etc) associated with smoking and drinking alcohol which can make a huge difference in the living standards. However, it is difficult to link the relation between acquiring the risk of gastric cancer with sex.

The standardized incidence of mortality from stomach cancer in men in Bulgaria is higher than the European average. Estimates for 2012 (http://eco.iarc.fr/EUCAN/) showed the incidence for Bulgaria at 21.4 per 100,000 men; while the average for Europe is 19.5 per 100000 men, and mortality rates were 17.9 and 14.6 per 100,000 men, respectively. In women the incidence of $(10.4$ per 100,000$)$ and mortality $(8.0$ per 100,000$)$ from stomach cancer in Bulgaria are also higher than 
the European average -9.3 and 7.0 per 100,000 women, respectively. ${ }^{13}$ In Bulgaria, the observed overall seropositivity of $H$. pylori-infection is more than $89 \% .^{21}$

In 2010, H. pylori eradication therapy for MALT lymphoma and early gastric cancer after endoscopic resection were approved by the Japanese health insurance system based on the results of a large-scale multicentre study (Japan Gast Study Group) in Japan published in 2008. In a systematic review, Shiota and co-workers ${ }^{22}$ have shown that $H$. pylori eradication therapy statistically diminished the prevalence of clinical gastric cancer by approximately a third, in Japan. Theoretically, complete eradication of $H$. pylori infections will prevent approximately 150,000 deaths from gastric cancer over the next 5 years. ${ }^{22}$

Based on this results of preliminary study of the possible risks of acquisition of infection and the development of gastric cancer in the region of the Stara Zagora, Bulgaria, all the first degree relatives of patients were informed for the higher risk for developing of gastric cancer and were directed to a gastroenterologist for clinical examinations and treatment of $H$. pylori- infection. Additionally, current work will be extended with more gastric cancer patients and their first degree relatives for more valid and concrete outcomes.

\section{Conclusions}

The prevalence of $H$. pylori is still high in the healthy asymptomatic population (over $80 \%$ ) in Bulgaria. The standardized incidence of and mortality from stomach cancer in people in Bulgaria is higher than the European average. Concern authorities should develop and implement strategies to improve educational status, basic sanitary facilities, and socioeconomic status to minimize $H$. pylori infection. We could recommend more active detection and treatment of infected children, which should result in specific and effective eradication of $H$. pylori, reduce the likelihood of transmission of infection and, as a rule, lead to a reduction in the incidence of gastric cancer in adults.

\section{Ethical approval}

Not required.

\section{Conflicts of interest}

The authors have none to declare.

\section{References}

1. Patel A, Shah N, Prajapati JB. Clinical application of probiotics in the treatment of
Helicobacter pylori infection-A brief review. J Microbiol Immunol Infect. 2014;47(5):429-437.

2. Hasni SA. Role of Helicobacter pylori infection in autoimmune diseases. Curr Opin Rheumatol. 2012;24:429-434.

3. Eshraghian A, Hashemi SA, Hamidian Jahromi A, et al. Helicobacter pylori infection as a risk factor for insulin resistance. Dig Dis Sci. 2009;54:1966-1970.

4. Gunji T, Matsuhashi N, Sato H, et al. Helicobacter pylori infection is significantly associated with metabolic syndrome in the Japanese population. Am J Gastroenterol. 2008;103:3005-3010.

5. IARC Working Group on the evaluation of carcinogenic risks to humans, Lyon, 7-14 June 1994. IARC Monogr Eval Carcinog Risks Hum. 1994;61:1-241.

6. Chan AOO, Wong B. Epidemiology of gastric cancer. http://www. up to date. com/ contents/epidemiology-of-gastric-cancer? source $=$ see link (accessed 12.08.15).

7. Cabebe EC. Gastric cancer. http://emedicine. medscape. com/article/278744-overview\#a8 (accessed 12.08.15).

8. Rokkas T, Sechopoulos P, Pistiolas D, Margantinis G, Koukoulis G. Helicobacter pylori infection and gastric histology in first-degree relatives of gastric cancer patients: a meta analysis. Eur J Gastroenterol Hepatol. 2010;22(9):1128-1133.

9. Shin CM, Kim N, Yang HJ, et al. Stomach cancer risk in gastric cancer relatives: interaction between Helicobacter pylori infection and family history of gastric cancer for the risk of stomach cancer. J Clin Gastroenterol. 2010;44(2):e34-9.

10. den Hollander WJ, Holster IL, den Hoed CM, et al. Ethnicity is a strong predictor for Helicobacter pylori infection in young women in a multi-ethnic European city. $J$ Gastroenterol Hepatol. 2013;28:1705-1711.

11. Ozaydin N, Turkyilmaz SA, Cali S. Prevalence and risk factors of Helicobacter pylori in Turkey: a nationally-representative, cross-sectional, screening with the (1)(3)C-Urea breath test. BMC Pub Health. 2013;13:1215.

12. Eusebi LH, Zagari RM, Bazzoli F. Epidemiology of Helicobacter pylori infection. Helicobacter. 2014;19(Suppl. 1):1-5.

13. Cancer incidence in Bulgaria, 2012. vol. XXIII. Sofia: Bulgarian National Cancer Registry; 2014.

14. Cancer incidence in Bulgaria, 2013. vol. XXIV. Sofia: Bulgarian National Cancer Registry; 2015.

15. Eshraghian A. Epidemiology of Helicobacter pylori infection among the healthy population in Iran and countries of the Eastern Mediterranean Region: a systematic review of prevalence and risk factors. World $J$ Gastroenterol. 2014;20(46):17618-17625.

16. Mladenova I, Chakarova B, Grekova O, Panayotova M, Petrov D. A preliminary study of association between risk factors for Helicobacter pylori and Giardia intestinalis in Bulgaria. HELICOBACTER. vol. 21. 111 RIVER ST, HOBOKEN 07030-5774, NJ USA: WILEY-BLACKWELL; 2016:110-111.

17. Mladenova IA, Grekova O, Kirova D, Petrov D. The families of gastric carcinoma patients-very important target group in Stara Zagora, Bulgaria. HELICOBACTER. 22. 111 RIVER ST, HOBOKEN 07030-5774, NJ USA: WILEY; 2017.

18. Bertuccio P, Rosato V, Andreano A, et al. Dietary patterns and gastric cancer risk: a systematic review and meta-analysis. Ann Oncol. 2013;24:1450-1458.

19. Mladenova-Hristova I. The inhibitory effect of genus Lactobacillus on Helicobacter pylori infection. Trakia J Sci. 2013;4:299-303.

20. Mégraud F, Bessède E, Varon C. Helicobacter pylori infection and gastric carcinoma. Clin Microbiol Infect. 2015;21(November (11)):984-990.

21. Mladenova-Hristova I. Epidemiological investigations of $H$. pylori infection. PhD Thesis. Sofia 2000; 2000.

22. Shiota S, Murakawi K, Suzuki R, Fujioka T, Yamaoka Y. Helicobacter pylori infection in Japan. Expert Rev Gastroenterol Hepatol. 2013;7(1):35-40. 